\title{
Tunable telephoto: governable Fourier spectrum anamorphic scaling
}

\author{
Cristina M. Gómez-Sarabia, ${ }^{1}$ Enrique Tajahuerce, ${ }^{2}$ (D) And \\ JoRGE OJEDA-CASTAÑEdA ${ }^{3, *}$ (D) \\ ${ }^{1}$ Artes Digitales, Universidad de Guanajuato, Salamanca, Guanajuato 36885, Mexico \\ ${ }^{2}$ GROC.UJI, Department de Física, Universitat Jaume I, E12071 Castelló, Spain \\ ${ }^{3}$ Departamento de Electrónica, Universidad de Guanajuato, Salamanca, Guanajuato 36885, Mexico \\ *jojedacas@ugto.mx
}

\begin{abstract}
We discuss the Gaussian design of a device that controls the scale, in an anamorphic fashion, of Fraunhofer diffraction patterns. The device uses two pairs of varifocal cylindrical lenses. For spherical lenses, the optical powers can be predicted by using a previously known high-level solution. We emphasize the anamorphic capabilities, by considering the case $\mathrm{M}_{\mathrm{y}}=1$ / $\mathrm{M}_{\mathrm{x}}$. The proposed device does not introduce vignetting, and it does not alter the axial location of the Fraunhofer diffraction patterns. Since the composing elements work at fixed inter-lens separation, the device does not require mechanical compensation.
\end{abstract}

(C) 2021 Optical Society of America under the terms of the OSA Open Access Publishing Agreement

\section{Introduction}

As pointed out by Abbe [1], under coherent illumination, image formation can be described in terms of Fraunhofer diffraction patterns. The conditions for describing Fraunhofer diffraction patterns, as Fourier spectra, are well described by Born and Wolf [2]. Under noncoherent illumination, the conceptual use of the Fourier spectra is rightly credited to Duffieux [3].

It is convenient to distinguish the generation of virtual Fraunhofer diffraction patterns [4, 5], from the commonly described real Fraunhofer diffraction patterns, which are frequently associated to the setups formed with identical lenses, separated by their focal length [6-9].

For spatial filtering operations, it is quite useful to be able to modify the scale of the Fraunhofer diffraction patterns for coupling Fourier spectra with the physical size of the spatial filters. This scaling operation is commonly implemented by axially displacing the input, under spherical illumination. Trivially, this technique introduces vignetting effects [10]. It is relevant here to note that for reducing the overall length of the optical processor, some writers have suggested to use telephoto lenses [11, 12]. However, these proposed devices do not offer tunable magnification.

We note that other authors have recognized the usefulness of introducing anamorphic magnifications, when performing optical filtering techniques [13, 14]. For implementing anamorphic processors, the optical setups usually have rather complex lens arrangements [15].

On the other hand, there are several relevant efforts for incorporating varifocal lenses, for proposing novel optical systems [16-18]. Some of these optical systems do not require mechanical compensation; as for example when implementing tunable magnifications with zero throw $[19,20]$.

Here, we discuss a Gaussian design of a simple device, which can scale the Fraunhofer diffraction pattern of any input masks. We note that the scaling operation can be anamorphic, and that it does not alter the longitudinal position of the Fourier spectra. Hence, the proposed device can be useful for coupling the size of Fourier spectra to the size of several spatial filters. To our end, we follow a simple treatment, but we do recognize that if the lenses are spherical, then the optical powers can be predicted by using a previously known, high-level solution [16]. However, we believe that we are discussing a novel application.

As shown in Fig. 1(a), we consider a classical optical processor. The composing lenses have fixed optical powers, which are equal to $\mathrm{K}_{1}$. As depicted in Fig. 1(b), after the first lens, we 


\section{OSA CONTINUUM}

locate two varifocal lenses, $\Lambda_{2}$ and $\Lambda_{3}$, respectively. The varifocal pair scales up the Fraunhofer diffraction pattern. And in Fig. 1(c) we depict a scale down operation.

(a)

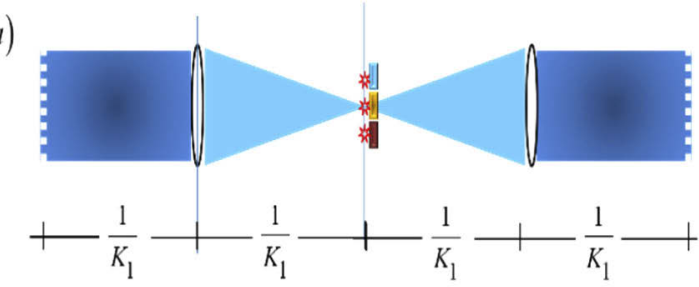

(b)

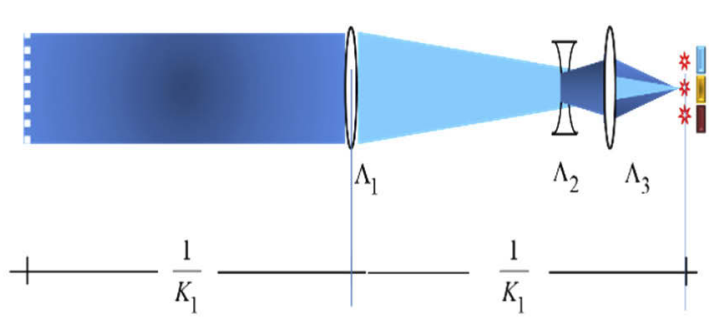

(c)

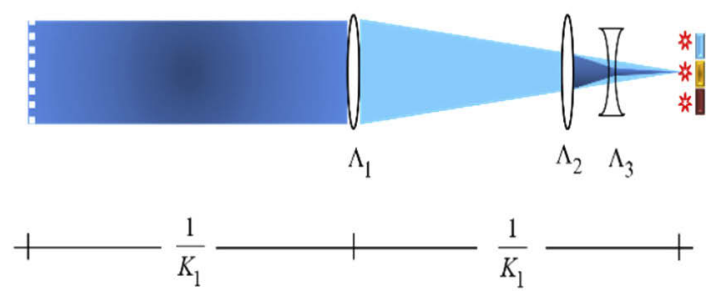

Fig. 1. Pictorial of a classical optical processor. In (a) an optical processing system with fixed optical powers. In (b) a scaling down operation on the Fourier spectrum. And in (c) a scaling up operation on the Fourier spectrum.

In section 2, we discuss the basics of the proposed device. In section 3 we specify the optical powers of the composing elements, along the horizontal and the vertical directions, for generating an anamorphic fitting operation. And in section 4, we summarize our contribution.

\section{Zero throw tunable fitting}

In Fig. 2, we depict the main paraxial variable of the proposed device. We consider a paraxial ray, parallel to optical axis, from the object to the first lens, $\Lambda_{1}$. If the height is $y_{1}$, then after refraction the paraxial angle reads

$$
u_{1}=-K_{1} y_{1}<0 .
$$

The selected paraxial ray impinges on the optical attachment, and after two consecutive refractions, it emerges with an angle

$$
u_{3}=\frac{u_{1}}{M}=-\frac{K_{1} y_{1}}{M} .
$$

In Eq. (2) we denote as M, the magnification needed for fitting the Fourier spectrum over a given spatial filter. 


\section{OSA CONTINUUM}

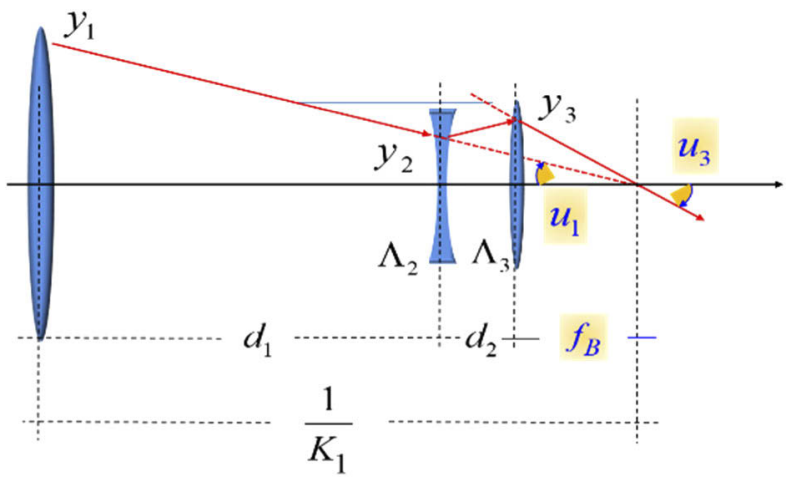

Fig. 2. Main paraxial variables describing the refraction at the two elements, $\Lambda_{2}$ and $\Lambda_{3}$ respectively, which compose the optical attachment.

It is apparent from Fig. 2 that

$$
f_{B}=\frac{1}{K_{1}}-\left(d_{1}+d_{2}\right) .
$$

Then, from Fig. 2 and from Eq. (3), we recognize that

$$
u_{3}=-\frac{y_{3}}{f_{B}}=\frac{y_{3}}{1-K_{1}\left(d_{1}+d_{2}\right)} K_{1} .
$$

We note that the heights, at the three elements are interrelated as follows

$$
y_{3}=M_{2} * y_{2}=M_{2} * M_{1} * y_{1}>0 .
$$

Consequently, from Eqs. (2), (4), and (5) we obtain

$$
M M_{1} * M_{2} *=1-K_{1}\left(d_{1}+d_{2}\right) .
$$

Now, as part of the upfront generic considerations, we recognize that the overall equivalent optical power has a rather simple expression. From Fig. 2, we notice that the exit paraxial angle $\mathrm{u}_{3}$ help us to relate the back focal length and the overall equivalent optical power. That is,

$$
\begin{gathered}
u_{3}=-K y_{1}=-M_{2} * M_{1} * \frac{y_{1}}{f_{B}} ; \\
K=\frac{K_{1}}{M} .
\end{gathered}
$$

From Eq. (7) we recognize that the zoom attachment modifies the overall equivalent optical power. And since the object is at star space, then the zoom attachment also modifies the telephoto ratio

$$
\frac{f_{B}}{f^{\prime}}=\frac{K}{K_{1}}=\frac{1}{M}
$$

As next discussed, the above relationships are sufficient for identifying the optical power of the zoom components, as functions of the variable magnification $\mathrm{M}$.

\section{Optical powers for anamorphic scaling}

For the sake of completeness in our discussion, in the appendix we summarized the approach in references [19] and [20]. However, we note that these results can be obtained by applying the 
generic high-level description in Ref. [16]. Here, it suffices to indicated that by using paraxial, ray tracing formulas and the condition on Eq. (6), one obtains the following optical powers, along the horizontal directions

$$
\begin{aligned}
& K_{2, x}=\left(1-\frac{1}{M_{x}}\right)\left(\frac{1}{d_{2}}\right) \frac{1-K_{1}\left(d_{1}+d_{2}\right)}{1-K_{1} d_{1}} . \\
& K_{3, x}=-\left(M_{x}-1\right)\left(\frac{1}{d_{2}}\right) \frac{1-K_{1} d_{1}}{1-K_{1}\left(d_{1}+d_{2}\right)} .
\end{aligned}
$$

In Fig. 3, we plot the Eqs. (7), (9) and (10) by setting $\mathrm{K}_{1}=0.05(1 / \mathrm{cm})$, that is $\mathrm{f}_{1}=20(\mathrm{~cm})$. And the inter-lens separations are $\mathrm{d}_{1}=7(\mathrm{~cm}) ; \mathrm{d}_{2}=7(\mathrm{~cm})$; and $\mathrm{f}_{\mathrm{B}}=6(\mathrm{~cm})$.

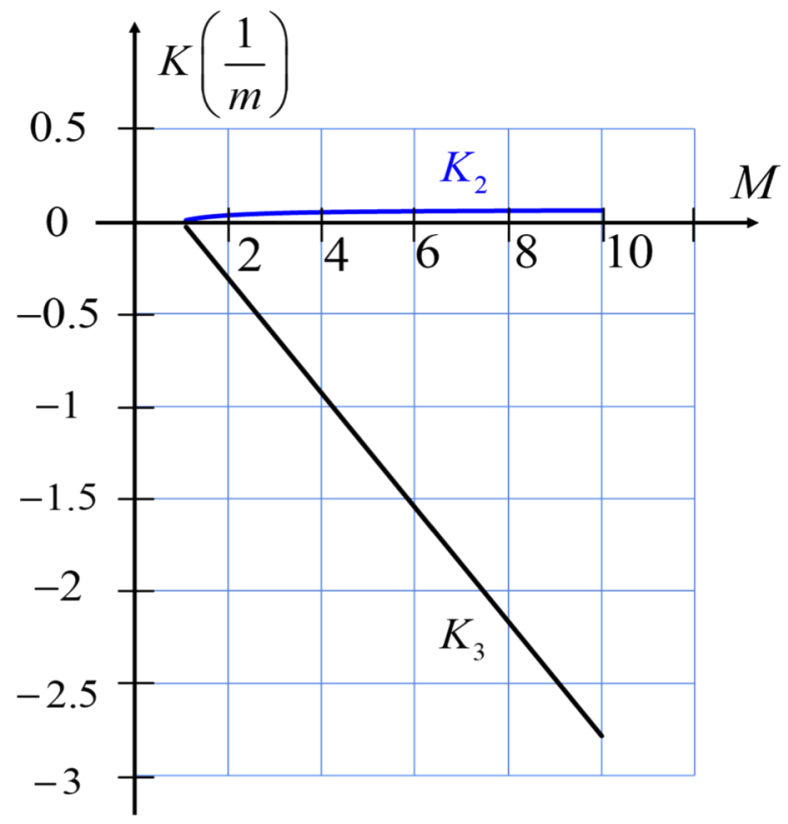

Fig. 3. Optical powers as function of the scaling magnification $\mathrm{M}$, in the interval $1.1 \mathrm{M}$ 10. We plot $K_{2}$ (in blue) and $K_{3}$ (in black), $K_{1}=0.05(1 / \mathrm{cm})$, then $f_{1}=20(\mathrm{~cm})$, and the inter-lens separations are $\mathrm{d}_{1}=7(\mathrm{~cm}) ; \mathrm{d}_{2}=7(\mathrm{~cm})$; and $\mathrm{f}_{\mathrm{B}}=6(\mathrm{~cm})$.

It is apparent from Fig. 3, that one can sustain a tunable scaling operation, in the interval $1.1 \leq \mathrm{M}_{\mathrm{x}} \leq 10$. For this operation, one needs an optical element with a moderate, positive hyperbolic variation $0.0(1 / \mathrm{cm}) \leq \mathrm{K}_{2, \mathrm{x}} \leq 0.06(1 / \mathrm{cm})$. And a second element with a linear, negative variation $-2.78(1 / \mathrm{cm}) \leq \mathrm{K}_{3, \mathrm{x}} \leq-0.03(1 / \mathrm{cm})$.

Then, we recognize that the proposed device is useful for continuously scaling (along the horizontal direction) the Fourier spectrum of an input picture, in the range $1.1 \leq \mathrm{Mx} \leq 10$; without altering the location of the Fraunhofer diffraction pattern.

For emphasizing the anamorphic characteristics of the proposed device, we only consider the following illustrative example, depicted in Fig. 4. Other cases are beyond our current scope. If we set

$$
M_{y}=\frac{1}{M_{x}} .
$$




\section{OSA CONTINUUM}

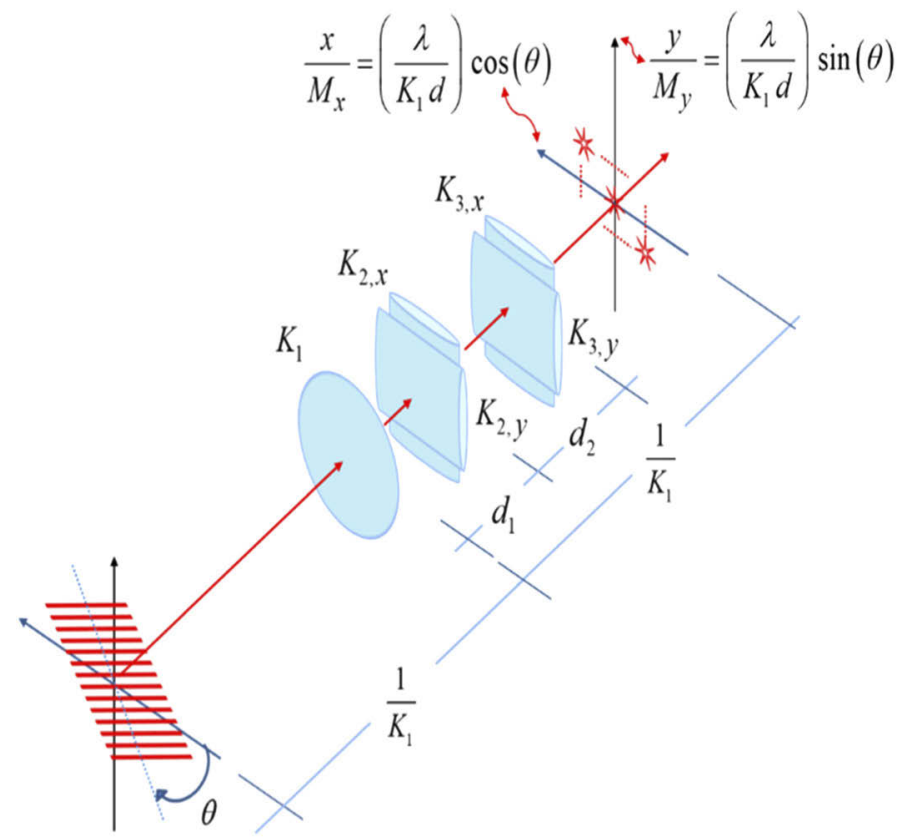

Fig. 4. Pictorial of a classical optical processor that uses one spherical lens, and two pairs of varifocal lenses. This device implements a tunable, anamorphic scaling operation on the Fourier spectrum.

Then, Eq. (9) becomes

$$
K_{2, y}=\left(1-M_{x}\right)\left(\frac{1}{d_{2}}\right) \frac{1-K_{1}\left(d_{1}+d_{2}\right)}{1-K_{1} d_{1}}
$$

And Eq. (10) becomes

$$
K_{3, y}=\left(1-\frac{1}{M_{x}}\right)\left(\frac{1}{d_{2}}\right) \frac{1-K_{1} d_{1}}{1-K_{1}\left(d_{1}+d_{2}\right)} .
$$

In Fig. 4, we assume that the input is an in-plane rotated (say by an angle $\theta$ ) cosinusoidal grating. Then the Cartesian spatial frequencies are

$$
\begin{aligned}
& \mu=\left(\frac{M_{x}}{\frac{\lambda}{K_{1}}}\right) x=\frac{\cos (\theta)}{d} ; \\
& \nu=\left(\frac{M_{y}}{\frac{\lambda}{K_{1}}}\right) y=\frac{\sin (\theta)}{d} .
\end{aligned}
$$

From Eq. (14) it is straightforward to obtain that

$$
x^{2}+y^{2}=\left(\frac{\lambda}{K_{1} d}\right)^{2}\left[\left(\frac{1}{M_{x}}\right)^{2}+\left(\frac{1}{M_{y}}\right)^{2}\right] .
$$

It is apparent, from Eq. (15), that the diffractions orders, of the Fourier spectrum, are now located along an ellipse. In general, the major axis and the minor axis depend on the horizontal magnification $\mathrm{M}_{\mathrm{x}}$, and the vertical magnification $\mathrm{M}_{\mathrm{y}}$, respectively. This is also true for the condition stated in Eq. (11). 


\section{Final remarks}

We have proposed the use of an optical device that controls the scale of the Fourier spectrum, without altering the axial location of the Fraunhofer diffraction pattern. For the scaling operation, we have incorporated the following requirements, related to the use of a classical optical processor. The input image is located at the front focal plane of an initial objective lens. And the Fourier spectrum should remain located, at the back focal plane of the initial objective lens.

We have discussed the Gaussian design of an optical device that works in conjunction with the initial objective lens. This device controls the scale of the Fourier spectrum, without altering the axial location of the Fraunhofer diffraction pattern.

The have proposed to employ two varifocal lenses, with fixed interlens separation. Thus, the device does not require of any mechanical compensation, while performing a continuous scaling operation, with zero throw.

By using a simple paraxial approach, we have identified the analytical expressions describing the optical powers, of the composing elements, as functions of the tunable magnification. However, we have noted that if the varifocal lenses are spherical, the optical powers can be derived from the higher-level treatment in reference [16].

We have recognized that even for large magnification values, the required optical powers have feasible values.

We have illustrated the anamorphic capabilities of the proposed device, by considering the variations of optical power with tunable magnification along the vertical axis and the horizontal axis.

\section{Appendix}

For obtaining the required optical powers, in Eqs. (9) and (10) in the main text, it is convenient to recognize that the input angle $\mathrm{u}_{1}$ is useful for identifying the first ratio $\mathrm{M}_{1} *$; that is

$$
\begin{gathered}
u_{1}=-K_{1} y_{1}=-\left(1-M_{1} *\right) \frac{y_{1}}{d_{1}} ; \\
M_{1} *=1-K_{1} d_{1} .
\end{gathered}
$$

By substituting Eq. (A1) in Eq. (6) we note that the second ratio reads

$$
M_{2} *=\left(\frac{1}{M}\right) \frac{1-K_{1}\left(d_{1}+d_{2}\right)}{1-K_{1} d_{1}} .
$$

Next, we note that the intermediate angle $\mathrm{u}_{2}$ satisfies the two following expressions

$$
\begin{gathered}
u_{2}=\frac{y_{3}-y_{2}}{d_{2}} ; \\
u_{2}=\frac{y_{2}-y_{1}}{d_{1}}-y_{2} K_{2} .
\end{gathered}
$$

By substituting the ratios $\mathrm{M}_{1} *$ and $\mathrm{M}_{2} *$ in Eq. (A3) we obtain

$$
\frac{\left(1-M_{2} *\right) M_{1} *}{d_{2}}=\frac{\left(1-M_{1} *\right)}{d_{1}}+M_{1} * K_{2} .
$$

And from Eq. (A4) we have that

$$
K_{2}=\frac{\left(1-M_{2} *\right)}{d_{2}}-\frac{\left(1-M_{1} *\right)}{M_{1} * d_{1}} .
$$


The result in Eq. (A5) is a full agreement with the result that can be obtained by applying Hopkinś procedure [20], which is restricted to fixed optical powers. For extending Hopkinś procedure is necessary to substitute Eqs. (A1) and (A2) in Eq. (A5). After doing so, we obtain the optical power $\mathrm{K}_{2}$, as in Eq. (9) in the main text.

Next, we identify the optical power of the third element. To that end, we note that the final angle $\mathrm{u}_{3}$ satisfies the two following expressions

$$
\begin{gathered}
u_{3}=-M_{2} * M_{1} * \frac{y_{1}}{f_{\text {Back }}} ; \\
u_{3}=-\frac{\left(1-M_{2} *\right) M_{1} * y_{1}}{d_{2}}-M_{2} * M_{1} * y_{1} K_{3} .
\end{gathered}
$$

From Eqs. (A1), (A2), and (A6) we obtain

$$
K_{3}=-\frac{\left(1-M_{2} *\right)}{M_{2} * d_{2}}+\frac{1}{f_{\text {Back }}} .
$$

Again, the result in Eq. (A7) is a full agreement with the result obtained by applying Hopkinś procedure [20]. And if one substitutes Eqs. (A1) and (A2) in Eq. (A7), we obtain the optical power $\mathrm{K}_{3}$, as reported in Eq. (10) in the main text.

Funding. Ministerio de Ciencia e Innovación, (PID2019-110927RB-I00); Generalitat Valenciana (PROMETEO/2020/029); Universitat Jaume I (UJIB2018-68).

Acknowledgments. Two authors (CMGS and JOC) are on sabbatical leave, at the Physics Department, Jaume I University, E12071 Castelló, Spain.

Disclosures. The authors declare that there are no conflicts of interest related to this article.

\section{References}

1. E. Abbe, "Beitrage zür Theorie des Mikroskops und fer Mikroskopischen wahrnehmung," Archiv. Mikroskopische Anat. 9(1), 413-468 (1873).

2. M. Duffieux, Ĺintégral de Fourier et ses applications à ĺoptique, pp. 79, 158 (Masson, Paris, 1970).

3. M. Born and E. Wolf, Principles of Optics, pp. 384-385 (Pergamon Press, Oxford, 1970).

4. S. G. Lipson and H. Lipson, Optical Physics, pp. 147 and 455 (Cambridge university press, Cambridge, 1969).

5. A. W. Lohmann, Optical information processing, Ed. S. Sinzinger, pp. 238-240 (Universitätsverlag, Ilmenau, 2006).

6. A. Marechal and M. Françon, Diffraction structure des images, p. 43 (Masson, Paris, 1970).

7. L. N. ÓNeill, Introduction to Statistical Optics, pp. 70-85 (Addison-Wesley, Reading, 1963).

8. A. Papoulis, Systems and transforms with applications in optics, p. 13 (McGraw-Hill, New York, 1968).

9. W. T. Cathey, Optical Information Processing and Holography, pp. 178-218 (John Wiley, New York, 1974).

10. J. W. Goodman, Introduction to Fourier optics, pp. 122-124 (McGraw-Hill, New York, 1988).

11. B. A. F. Blandford, "A new lens system for use in optical data processing," in Optical Instruments and Techniques, J. Home Dickson ed., 435-443 (Oriel press, Newcastle, 1970).

12. I. Juvells, S. Vallmitjana, and S. Bosch, "Analysis of a scalable tunable telephoto lens diffractometer and its use in optical correlation,” J. Mod. Opt. 39(5), 1107-1115 (1992).

13. N. F. Barber, "Diffraction Analysis of a Photograph of the Sea," Nature 164(4168), 485 (1949).

14. A. Kozma, E. N. Leith, and N. G. Massey, “Tilted-Plane Optical Processor,” Appl. Opt. 11(8), 1766-1777 (1972).

15. T. Szoplik and H. Arsenault, "Rotation-variant optical data processing using the 2-D, nonsymmetric Fourier transform," Appl. Opt. 24(2), 168-172 (1985).

16. A. Mikš and J. Novák, "Analysis of two-element zoom systems based on variable power lenses," Opt. Express 18(7), 6797-6809 (2010).

17. J. Ojeda-Castañeda, S. Ledesma, and C. M. Gómez-Sarabia, "Tunable apodizers and tunable focalizers using helical pairs," Photonics Lett. Pol. 5(1), 20-22 (2013).

18. S. Bernet, "Zoomable telescope by rotation of toroidal lenses," Appl. Opt. 57(27), 8087-8095 (2018).

19. C. M. Gómez-Sarabia and J. Ojeda-Castaneda, "Hopkins procedure for tunable magnification: surgical spectacles," Appl. Opt. 59(13), D59-D63 (2020).

20. Cristina M. Gómez-Sarabia and Jorge Ojeda-CastaÑeda, "Two-conjugate zoom system: the zero-throw advantage," Appl. Opt. 59(23), 7099-7102 (2020). 